\title{
Phosphoinositide 3-kinase inhibitors are effective therapeutic drugs for the treatment of hepatocellular carcinoma?
}

\author{
Jeong Su Park and Soo Han Bae \\ Severance Biomedical Science Institute, Yonsei University College of Medicine, Seoul, Korea
}

Keywords: Carcinoma, Hepatocellular; PI3K inhibitor; GSK3ß; EMT

Dear Editor,

Recently, we read an article entitled "Inhibition of PI3K/Akt signaling suppresses epithelial-to-mesenchymal transition in hepatocellular carcinoma through the Snail/GSK-3/beta-catenin pathway" by Lee et al.' with profound interest. The study showed that phosphoinositide 3-kinase (PI3K) inhibitors, LY294002 and Idelalisib, restrict epithelial-to-mesenchymal transition as indicated by upregulation of $\mathrm{E}$-cadherin and downregulation of $\mathrm{N}$-cadherin. Furthermore, PI3K inhibitors were reported to induce nuclear translocation of GSK-3 $\beta$ that was shown to decrease the expression of Snail and $\beta$-catenin in HepG2 and Huh-BAT cells. Subsequently, PI3K inhibitors were reported to supress the proliferation and invasion of hepatocellular carcinoma (HCC). However, before interpreting the results of the published study, several concerns should be taken into consideration.

At first, class I PI3K signaling has been known to be crucial for HCC progression, and thereby, substitutes as a therapeutic target for the treatment of HCC. Furthermore, PI3K has three catalytic subunits, which includes PI3K- $a$, PI3K- $\beta$, PI3K- $\delta .{ }^{2}$ Several studies have reported that upregulation of PI3K $\delta$ is positively correlated with progression of human advanced cancers such as HCC and melanoma. ${ }^{3,4}$ Moreover, Idelalisib is well-known as a first-in-class PI3K $\delta$ inhibitor for the treatment of leukemia. ${ }^{5}$ Recently, it has been reported that Idelalisib suppresses HCC progression. ${ }^{4}$ Subsequently, high expression levels of PI3K $\delta$ have been associated with advanced $\mathrm{HCC}{ }^{4}$ However, PI3K $\delta$ expression levels in Huh7BAT and HepG2 cell lines have not been shown in this study. It is crucial to elucidate the effects of PI3K $\delta$ inhibitor on HCC progression.

Secondly, it is well-known that LY294002 inhibits PI3K/AKT/ mammalian target of rapamycin complex 1 (mTORC1) pathway. ${ }^{6}$ In several cancers, this has been reported to cause significant reduction in the capability of cancer cells pertaining to viability, migration, and invasion. ${ }^{7.8}$ However, AKT phosphorlation has been shown to be upregulated in LY294002-treated Huh-BAT cells. Thus, attributes of Huh-BAT cells need to be elucidated. Furthermore, the detailed mechanisms of LY294002 that lead to inhibi-

\begin{abstract}
Abbreviations:
GSK3 3 , glycogen synthase kinase 3; HCC, hepatocellular carcinoma; mTORC1, mammalian target of rapamycin complex 1; PI3K, phosphoinositide 3-kinase; TGF, transforming growth factor
\end{abstract}

\section{Corresponding author : Soo Han Bae}

Severance Biomedical Science Institute, Yonsei Biomedical Research Institute, Yonsei University College of Medicine, 50-1 Yonsei-ro, Seodaemun-gu, Seoul 03722, Korea

Tel: +82-2 2228-0756, Fax: +82-2-2227-8129

E-mail:soohanbae@yuhs.ac

http://orcid.org/0000-0002-8007-2906 
tion of PI3K/AKT/mTORC1 pathway in HCC cell lines are not yet evident. Subsequently, the mechanism needs to be further validated.

Furthermore, diverse roles of serine/threonine kinase glycogen synthase kinase 3 (GSK3 3 ) in cancer progression still remain controversial. ${ }^{6}$ However, several studies have reported that GSK3 $\beta$ acts as a tumor suppressor gene, and thereby, it has been suggested to be a promising therapeutic target for $\mathrm{HCC}$ treatment. ${ }^{9-11}$ Additionally, GSK3 $\beta$ has been associated with signaling pathways such as Notch-, Wnt/ $\beta$-catenin-, and transforming growth factor (TGF) $\beta$-pathway, that have been implicated in HCC pathogenesis. ${ }^{12}$ However, the detailed interactive mechanisms of GSK3 $\beta$ and PI3K inhibitors are not elucidated. This study showed that PI3K inhibitors upregulate the expression of nuclear GSK3 $\beta$, which further suppresses snail expression in liver cancer cell lines.

In conclusion, Lee et al.'s study' provides the anti-cancer effects and comprehensive mechanisms of PI3K inhibitors in HCC cell lines. It suggests that PI3K is a potential therapeutic target for the treatment of HCC.

\section{Authors' contribution}

Jeong Su Park: manuscript writing and critical revision; Soo Han Bae: critical revision and supervision

\section{Conflicts of Interest}

The authors have no conflict to disclose.

\section{REFERENCES}

1. Lee $S$, Choi EJ, Lee YB, Lee JH, Yu SJ, Yoon JH, et al. Inhibition of $\mathrm{PI3K} / \mathrm{Akt}$ signaling suppresses epithelial-to-mesenchymal transition in hepatocellular carcinoma through the Snail/GSK-3/beta-catenin pathway. Clin Mol Hepatol 2020;26:529-539.
2. Fruman DA, Chiu H, Hopkins BD, Bagrodia S, Cantley LC, Abraham RT. The PI3K pathway in human disease. Cell 2017;170:605-635.

3. Ali K, Soond DR, Pineiro R, Hagemann T, Pearce W, Lim EL, et al. Inactivation of $\mathrm{PI}(3) \mathrm{K}$ p110 $\mathrm{\delta}$ breaks regulatory T-cell-mediated immune tolerance to cancer. Nature 2014;510:407-411.

4. Ko E, Seo HW, Jung ES, Ju S, Kim BH, Cho H, et al. PI3KS is a therapeutic target in hepatocellular carcinoma. Hepatology 2018;68: 2285-2300.

5. Yang Q, Modi P, Newcomb T, Quéva C, Gandhi V. Idelalisib: first-inclass PI3K delta inhibitor for the treatment of chronic lymphocytic leukemia, small lymphocytic leukemia, and follicular lymphoma. Clin Cancer Res 2015;21:1537-1542.

6. Patel S, Woodgett J. Glycogen synthase kinase-3 and cancer: good cop, bad cop? Cancer Cell 2008;14:351-353.

7. Xu F, Na L, Li Y, Chen L. Roles of the PI3K/AKT/mTOR signalling pathways in neurodegenerative diseases and tumours. Cell Biosci 2020;10:54.

8. Ma J, Xie SL, Geng YJ, Jin S, Wang GY, Lv GY. In vitro regulation of hepatocellular carcinoma cell viability, apoptosis, invasion, and AEG-1 expression by LY294002. Clin Res Hepatol Gastroenterol 2014;38:73-80.

9. Huang KT, Huang YH, Li P, He B, Chen ZK, Yu X, et al. Correlation between tuberous sclerosis complex 2 and glycogen synthase kinase 3 beta levels, and outcomes of patients with hepatocellular carcinoma treated by hepatectomy. Hepatol Res 2014;44:1142-1150.

10. Chua HH, Tsuei DJ, Lee PH, Jeng YM, Lu J, Wu JF, et al. RBMY, a novel inhibitor of glycogen synthase kinase $3 \beta$, increases tumor stemness and predicts poor prognosis of hepatocellular carcinoma. Hepatology 2015;62:1480-1496.

11. Zhang N, Liu X, Liu L, Deng Z, Zeng Q, Pang W, et al. Glycogen synthase kinase-3 $\beta$ inhibition promotes lysosome-dependent degradation of c-FLIPL in hepatocellular carcinoma. Cell Death Dis 2018;9:230.11.

12. Cervello M, Augello G, Cusimano A, Emma MR, Balasus D, Azzolina $A$, et al. Pivotal roles of glycogen synthase-3 in hepatocellular carcinoma. Adv Biol Regul 2017;65:59-76. 